\title{
ESTILOS PARENTAIS E CONSUMO DE DROGAS ENTRE ADOLESCENTES: REVISÃO SISTEMÁTICA
}

\author{
Fernando Santana de Paiva* \\ Telmo Mota Ronzani ${ }^{\#}$
}

\begin{abstract}
RESUMO. Na tentativa de compreender o consumo abusivo de álcool e outras drogas durante a adolescência, inúmeras pesquisas têm apontado a família como um importante fator de risco ou proteção. Diante disso, o presente artigo teve por objetivo realizar uma revisão sistemática a respeito de trabalhos científicos sobre a associação entre os estilos e práticas parentais de socialização e o consumo de substâncias psicoativas entre adolescentes. Os artigos foram pesquisados em três bancos de dados - Medline, Adolec e Pubmed, sendo utilizados dois descritores de busca bibliográfica: parental styles e parental monitoring juntamente com substance use. A amostra final se constituiu de 30 artigos, provenientes de diferentes países. Através dos resultados deste trabalho, foi possível constatar uma significativa associação entre o consumo abusivo de álcool e outras drogas na adolescência e os estilos e práticas parentais. Estes achados apontam a importância de se conhecer essa relação no contexto brasileiro.
\end{abstract}

Palavras-chave: estilos parentais, adolescência, substâncias psicoativas.

\section{PARENTAL STYLES AND CONSUMPTION OF DRUGS AMONG ADOLESCENTS}

\begin{abstract}
In order to understand alcohol and other drugs abusive consumption during adolescence, several researches have pointed out the family as an important risk or protection factor. This study aims to accomplish a systematic review of scientific papers regarding the association between parental styles and socialization practices and psychoactive substances consumption among adolescents. The articles were researched in three databases - Medline, Adolec and Pubmed -, using two terms in order to restrict the bibliographic search: parental styles and parental monitoring together with substance use. The final sample was constituted of 30 papers from different countries. We could verify a significant association between alcohol and other drugs abusive consumption in adolescence and parental styles and practices. Such findings demonstrate the importance of getting to know this relationship in the Brazilian context.
\end{abstract}

Key words: Parental styles, adolescence, psychoactive substances.

\section{ESTILOS PARENTALES Y EL CONSUMO DE DROGAS ENTRE ADOLESCENTES}

RESUMEN. En el intento de comprenderse el consumo abusivo de alcohol y otras drogas durante la adolescencia, innúmeras investigaciones apuntan la familia como un importante factor de riesgo o protección. Así, el presente artículo tuvo como objetivo realizar una revisión sistemática sobre trabajos científicos relativos a la asociación entre los estilos y prácticas parentales de socialización y el consumo de sustancias psicoactivas entre adolescentes. Los artículos fueran investigados en tres bancos de datos - Medline, Adolec y Pubmed, siendo utilizados dos descriptores en la búsqueda bibliográfica: parental styles y parental monitoring juntamente con substance use. La muestra final fue constituida por 30 artículos provenientes de diferentes países. A través de los resultados de este trabajo, fue posible constatar una significativa asociación entre el consumo abusivo de alcohol y otras drogas en la adolescencia y los estilos y prácticas parentales. Estos hallazgos apuntan la importancia de conocer esta relación en el contexto brasileño.

Palabras-clave: Estilos parentales, adolescencia, substancias psicoativas.

* Psicólogo e Mestrando em Saúde Coletiva pela Universidade Federal de Juiz de Fora.

\# Doutor em Ciências da Saúde. Professor Adjunto do Departamento de Psicologia da Universidade Federal de Juiz de Fora, Programa de Pós-Graduação em Psicologia, Pólo de Pesquisa em Psicologia Social e Saúde Coletiva-POPSS. 
Ao longo dos últimos anos, inúmeros estudos têm procurado compreender o impacto que os pais exercem no desenvolvimento sociocognitivo dos filhos (Baurimd, 1971; Maccoby \& Martin,1983; Darling \& Steinberg, 1993; Alvarenga \& Piccinini, 2001) e na promoção de sua saúde mental (Li, Stanton \& Feigelman, 2000; Verdurmen, Monshouuwer, Dorsselaer, Bogt \& Vollebergh, 2005). Estes e outros pesquisadores têm procurado entender como a adoção de diferentes estilos parentais pode influenciar de maneira geral o desenvolvimento dos filhos (Baker 1999; Jackson, Henriksen, Dickinson, Messer \& Robertson, 1998; Jackson, Henriksen \& Dickinson, 1999; Mott, Crowe, Richardson \& Flay , 1999; Raboteg-Saric, Rijavec \& Brajsa-Zganec, 2001).

Os estilos parentais tiveram seus primeiros estudos com Baumrind (1971), que integrou tanto os aspectos comportamentais quanto os afetivos envolvidos na criação dos filhos. Essa autora propôs três estilos parentais: "com autoridade" (authoritative), autoritário e permissivo. Na década de 1980 MacCoby e Martin (1983), a partir dos estudos de Baumrind (1971), definiram os estilos parentais em função de duas dimensões subjacentes: a exigência (controle) e o apoio (afeto). Os estilos parentais que surgem como resultado desta combinação são os mesmos propostos por Baumrind (1971), com a diferença de que o estilo permissivo foi desmembrado em dois: o negligente e o indulgente. Assim, pais e mães autoritários possuem muita exigência e pouco afeto, os "com autoridade" fazem muita exigência e ao mesmo tempo são envolvidos afetivamente com seus filhos, os indulgentes estabelecem pouco controle e são muito afetivos e, por último, pais e mães negligentes são permissivos e indiferentes, com baixo grau de controle e afeto para com os filhos.

Autores como Darling e Steinberg (1993) ainda registram a diferença entre estilos parentais e práticas educativas. Pode-se considerar que os estilos parentais referem-se a um padrão global de características da interação dos pais com os filhos em diversas situações, um conjunto de atitudes que propicia um clima emocional em que as práticas se expressam.

O estilo então pode ser entendido como o conjunto de determinadas condutas. Desta forma, o estilo e a prática educativa estão normalmente associados, uma vez que o conjunto das práticas vai formar o estilo parental (Tornaría, Vandemeulebroecke \& Copin, 2001). São chamadas de práticas educativas as estratégias e técnicas empregadas por pais e mães no sentido de orientar o comportamento de seus filhos, buscando alcançar objetivos específicos em determinadas situações. São também denominadas por alguns autores de estratégias educativas ou de socialização, uma vez que têm como função comunicar à criança a vontade dos pais de que ela altere seu comportamento ou que se comporte de uma determinada maneira (Cecconello, Antoni \& Koller, 2003; Alvarenga \& Piccinini, 2001; Darling \& Steinberg, 1993).

\section{OS ESTILOS PARENTAIS E O CONSUMO DE ÁLCOOL E DROGAS ENTRE ADOLESCENTES}

Estudos epidemiológicos têm mostrado que o início do consumo de álcool, cigarro e outras drogas ocorre predominantemente durante a adolescência (Laranjeira, Pinsky, Zaleski \& Caetano, 2007; Carlini, Galduróz, Noto \& Nappo, 2005). A configuração de um ambiente favorável à adoção de comportamentos prejudiciais à saúde pelos jovens, como o consumo de substâncias psicoativas, é influenciada por uma série de fatores, sendo a família um dos mais importantes (Guo, Hawkins, Hill \& Abbott, 2001; Organização Mundial de Saúde [OMS], 2004; Schenker \& Minayo, 2005; Shope, Waller, Trivellore, Raghunathan \& Patil, 2001; Simons-Mortons, 2002).

A instituição familiar é considerada um dos elos mais fortes dessa cadeia multifacetada que pode levar ao uso abusivo de álcool e drogas, além de também atuar como importante fator de proteção. Isto se explica pelo fato de que os diferentes comportamentos sociais, entre eles o consumo de substâncias psicoativas, são aprendidos, predominantemente, a partir das interações estabelecidas entre o jovem e suas fontes primárias de socialização, que no Ocidente são a família, a escola e o grupo de amigos. O resultado desta constante interação é o estabelecimento de um vínculo que possibilita a comunicação de um conjunto de normas. Dificuldades percebidas nestas interações sociais podem se configurar em sérios fatores de risco para o surgimento de problemas na vida dos adolescentes (Schenker \& Minayo, 2003).

A falta de suporte parental, o uso de drogas pelos próprios pais, atitudes permissivas dos pais perante o consumo e incapacidade dos pais de controlar os filhos são fatores predisponentes à 
iniciação ou continuação de uso de drogas (Bahr, Hofmann \& Yang, 2005). Nesta perspectiva, estudos empíricos têm apontado que os diferentes estilos parentais de socialização e as práticas educativas que permeiam a relação entre pais e filhos funcionam como variáveis psicossociais capazes de exercer grande influência na adoção de diferentes comportamentos prejudiciais à saúde entre os jovens, entre eles o consumo de substâncias psicoativas (Duncan, Duncan, Biglan \& Ary, 1998; Rai et al., 2003; Ramirez, Crano, Quist, Burgoon, Álvaro \& Grandpre, 2004; SimonsMorton, Chen, Abroms \& Haynie, 2004; Wright \& Fitzpatrick, 2004;). O objetivo do presente artigo é identificar trabalhos de pesquisa e resultados de estudos empíricos que buscaram avaliar a associação entre o consumo de substâncias psicoativas e os estilos e práticas parentais de socialização.

\section{METODOLOGIA}

Primeiramente procedeu-se ao processo de busca de artigos a partir dos descritores bibliográficos Parental monitoring, Parental styles e substance use. As bases de dados definidas para a busca foram o Medline, Pubmed e Adolec no período de janeiro de 1997 a julho de 2007. Num segundo momento, procedeu-se ao processo de inclusão e exclusão dos artigos. O descritor monitoramento parental e consumo de substâncias apresentou o maior número de referências - 337, ao todo - enquanto estilos parentais apresentaram apenas 52 referências. Foram mantidos somente os artigos de natureza empírica sobre a associação, a influência e o impacto dos diferentes estilos e práticas de socialização parental no consumo de substâncias psicoativas durante a adolescência.

A partir da leitura acurada dos seus resumos, foram incluídos aqueles estudos que preenchiam os seguintes critérios:

a) apresentar como delineamento do tipo de pesquisa longitudinal, Survey ou caso-controle;

b) apresentar objetivos, métodos e resultados claramente definidos no resumo de cada texto;

c) a pesquisa ter sido realizada apenas com indivíduos entre 10 e 21 anos.

Foram excluídos trabalhos com uma um mais das seguintes características: a) que fossem teses, dissertações, revisões, intervenções e materiais educativos;

b) em que não houvesse uma descrição metodológica completa (objetivos, métodos e resultados);

c) que fossem estudos realizados com pais e outros membros familiares.

Nos três bancos de dados foram identificados 55 artigos que preencheram os critérios de inclusão e exclusão, porém 25 deles estavam presentes tanto no Medline quanto no Adolec. Destarte, ao final da definição de inclusão e exclusão dos artigos encontrados na primeira etapa, foram selecionados 30 artigos para a terceira etapa. A partir daí foi realizada a tabulação dos estudos encontrados, com discriminação dos seguintes itens: autores; ano; descrição da amostra e análise dos resultados encontrados. Finalmente, realizou-se a análise descritiva e qualitativa da amostra bibliográfica, acompanhada de discussão crítica do material.

\section{RESULTADOS}

Os 30 estudos da amostra final constituem trabalhos de pesquisa originais, assim distribuídos segundo o ano de publicação: uma publicação em 1997; duas em 1998; três em 1999; uma em 2000; quatro em 2001; duas em 2002; três em 2003; seis em 2004; seis em 2005 e duas em 2006. O interesse em compreender a relação entre o uso de substâncias e os estilos parentais demonstrou um crescimento desde a metade dos anos 90, aumentado ainda mais no início deste século. Os países que mais desenvolveram estudos sobre o tema foram os Estados Unidos da América (EUA), com 25 artigos, e a Inglaterra, com três artigos. Quanto ao tipo de estudo empírico realizado, as pesquisas de delineamento longitudinal e do tipo Survey foram as mais empregadas, sendo a duração dos estudos heterogênea. Nos estudos longitudinais os períodos de acompanhamento variaram de seis meses a onze anos. No caso dos estudos do tipo Survey, observou-se que grande parte foi realizada no âmbito escolar. No que se refere ao tamanho e tipo de amostra estudada, elas também diferem, variando o número dos participantes de 176 até 4.230 jovens. Apenas uma pesquisa foi realizada exclusivamente com adolescentes do sexo feminino, ao passo que todos os outros estudos se propuseram a pesquisar jovens de ambos os sexos. 
Tabela 1. Caracterização das Categorias de Análise das Pesquisas Realizadas e Principais Resultados Encontrados em Revisão Sistemática de Literatura nos Bancos de dados Medline, Adolec e Pubmed entre janeiro de 1997 e julho de 2007.

\begin{tabular}{ll}
\hline Categorias & Resultados \\
\hline Estilos Parentais & \\
"com autoridade" e não permissivo & Menor consumo de álcool, tabaco e outras drogas \\
Negligente & Maior consumo de álcool, tabaco e outras drogas
\end{tabular}

Práticas Parentais

Monitoramento

Supervisão

Comunicação

Suporte

Envolvimento

\section{Outros fatores analisados}

Uso de drogas pelos irmãos

Expectativas e atitudes parentais positivas

Uso de álcool e tabaco por pais e amigos

Papéis familiares claros

Bom desempenho acadêmico

Participação em atividades sócio-comunitárias
Menores índices de prevalência de uso de álcool, tabaco e outras drogas Fatores de proteção contra o uso de drogas
Os resultados apontam que os adolescentes que recebem maior monitoramento parental são os que apresentam menores taxas de envolvimento com drogas (Springer, Sharma, Guardado, Nava \& Kelder, 2006; Borawski, Ievers-Landis, Lovegreen \& Trapl, 2003; DiClemente et al., 2001). Práticas parentais como procurar localizar seus filhos, estar atento às suas atividades, saber quem são seus amigos, o que eles fazem no tempo livre e como eles gastam seu dinheiro são consideradas medidas que representam esforços parentais para monitorar e vigiar, ou seja, estão ligadas ao conhecimento parental sobre os filhos (Stattin \& Kerr, 2000). Os estudos apontaram que o monitoramento foi percebido como uma prática parental muito significativa para o menor consumo de álcool, cigarro e outras drogas entre os adolescentes.

Outras duas práticas parentais que também apresentaram resultados significativos contra o consumo de drogas foram o suporte e o envolvimento parental. Elas estão relacionadas à capacidade dos pais em responder às demandas dos filhos, sendo presentes de maneira mais construtiva entre os pais que adotam um estilo parental "com autoridade", os quais propiciam um clima emocional que transmite aos seus filhos o apoio necessário para a resolução dos problemas por eles vivenciados. A ausência de suporte parental pode colocar o adolescente sob maior vulnerabilidade para o uso abusivo de drogas, especialmente porque aumenta a probabilidade de envolvimento com pares usuários (Bahr et al., 2005; Hill, Hawkins, Catalano, Abbott \& Guo, 2005; Nash,
McQueen \& Bray, 2005; Wills, Resko, Ainette \& Mendoza, 2004). Alguns resultados apontaram especialmente a importância de uma adequada comunicação entre pais e filhos e as expectativas dos pais com relação ao uso de álcool como fatores determinantes para o não-consumo entre os jovens. Dessa forma, o conhecimento sobre as expectativas parentais a respeito do uso de drogas pode funcionar como proteção entre os jovens, daí a importância de um estilo parental que promova um clima emocional propício a esta transmissão de valores, pois aumentará as chances de apreensão por parte dos adolescentes (Darling \& Steinberg, 1993). Este conjunto de práticas parentais adotadas - monitoramento, suporte, e comunicação - está associado aos diferentes estilos empregados pelos pais. Assim, os estilos parentais "com autoridade" e não permissivo foram relacionados com o menor consumo de álcool e drogas entre os adolescentes, diferentemente dos resultados encontrados entre os pais negligentes (Shucksmith, Glendinning \& Hendry, 1997).

Além da família, os estudos analisados se preocuparam bastante em compreender o impacto do grupo de pares no uso abusivo de drogas. Durante muito tempo, essa variável foi percebida como a principal influência no tocante à questão das drogas, porém os estudos indicaram que a relação estabelecida com a família pode ser muito mais determinante. Alguns estudos pesquisaram a capacidade da família de minimizar o efeito de grupo de pares desviantes. $\mathrm{O}$ conjunto de práticas não permissivas, a definição clara 
de papéis familiares e principalmente o monitoramento e as expectativas negativas dos pais quanto ao uso de álcool minimizaram o efeito do grupo de amigos usuários (Wood, Read, Mitchell \& Brand, 2004; Stanton et al., 2002; Reifman, Barnes, Dintcheff, Farrel \& Uhteg, 1998).

\section{DISCUSSÃO}

O uso de substâncias psicoativas, por diferentes aspectos, é apontado por instituições e grupos sociais como um grave problema de saúde pública. Considerando-se o período estabelecido para esta revisão, nos três bancos de dados pesquisados foi possível encontrar que o consumo de substâncias psicoativas está marcadamente influenciado pelas diferentes experiências psicossociais com as quais o jovem se depara (Simons-Morton \& Haynie, 2003; Simons-Morton, 2004; Chuang, Ennet, Bauman \& Foshee, 2005; Clevend, Gibbons, Gerrard, Pomery \& Brody, 2005; Siebenbruner, Englund, Egeland \& Hudsons, 2006).

A relação com a família, a pressão do grupo de amigos, o desempenho acadêmico e a ligação com a escola, a inserção e incorporação de valores comunitários, a capacidade de decodificação de mensagens provenientes da mídia, além de aspectos individuais como auto-estima, capacidade de resolução de problemas e expectativas funcionais com relação ao uso de álcool e outras drogas são aspectos fundamentais para a compreensão do desenvolvimento de comportamentos prejudiciais à saúde entre os jovens, entre eles o consumo de álcool e outras drogas (Schenker \& Minayo, 2005).

A partir dos resultados deste trabalho pôde-se notar que não basta o desenvolvimento de estratégias pautadas por uma ótica exclusivamente individual, que contemple apenas o adolescente. Os artigos analisados apontam que a influência da família foi significativamente encontrada em diversos contextos e populações pesquisados. Os diferentes estilos parentais de socialização - "com autoridade”, autoritário, negligente e indulgente (Maccoby \& Martin, 1983; Darling \& Steinberg, 1993) - por meio das práticas educativas deles decorrentes foram amplamente relacionados com uso de substâncias psicoativas. Portanto, ao se pensar em ações relacionadas ao consumo de substâncias psicoativas, a família deve ser considerada. Reforçar seus vínculos e contribuir para que os pais adotem posturas mais benéficas para com os filhos, a partir de práticas mais positivas de socialização, podem produzir resultados mais eficazes. A compreensão do papel das práticas parentais pode contribuir para que pais estejam mais conscientes do seu papel na consolidação de crenças, valores e atitudes contrárias a comportamentos prejudiciais à saúde e ao desenvolvimento mental de seus filhos. Os jovens que têm maior apoio e suporte e se sentem compreendidos pela família apresentam menor padrão do consumo de drogas. Os resultados indicam ainda que o afeto e o interesse mostrados pelos pais, o tempo que passam com seus filhos e a firmeza de medidas disciplinares mantêm a relação com a abstenção do uso de drogas.

No Brasil, os esforços dirigidos para o entendimento dos aspectos psicossociais envolvidos no uso abusivo de álcool e outras drogas entre os jovens poderão contribuir para o fortalecimento de políticas de saúde e educação que pretendam concorrer para a promoção de uma vida mais saudável entre adolescentes e pais, bem como ampliar os conhecimentos de diferentes profissionais que lidam, cotidianamente, com os desafios impostos pela questão das drogas em nossa sociedade. A presente pesquisa demonstra a importância dos estilos parentais de socialização entre adolescentes como fator de proteção para o uso de álcool e outras drogas em diversos países. Tais resultados ressaltam a importância da avaliação empírica deste aspecto para a população brasileira. Em função da realidade epidemiológica do uso de substâncias psicoativas entre jovens brasileiros, tais informações tornam-se importantes para a formulação de ações de prevenção em nosso país.

\section{REFERÊNCIAS}

Baumrind, D. (1971). Harmonious parents and their preschool children. Developmental Psichology, 4(1), 99-102.

Maccoby, E., \& Martin, J. (1983). Socialization in the context of the family: Parent-child interaction. In E. M. Heteringhton (Ed.), P. H. Mussen (Org. Série), Handbook of child psychology ( Vol. 4: Socialization, personality, and socil development, ( $4^{\mathrm{a}}$ ed., pp. 1101). New York: Wiley.

Darling, N. \& Steinberg, L. (1993). Parenting Style as Context: an integrative Model. Psychological Bulletin, 113(3), 487-496.

Alvarenga, P. \& Piccininni, C. (2001). Práticas Educativas Maternas e Problemas de Comportamento em Pré-Escolares. Psicologia: Reflexão e Crítica, 14(3), 449-460.

Verdurmen, J., Monshouuwer, K., Dorsselaer, S., Bogt, T., \& Vollebergh, W. (2005). Alcohol Use and Mental Health in Adolescents: Interactions with Age and Gender-Findings from the Dutch 2001 Health Behaviour in School-Aged Children Survey. Journal of studies on Alcohol, 66(5), 605609.

Tornaría, M. L. G., Vandemeulebroecke, L., \& Colpin, H. (2001). Pedagogia familiar. Montevideo: Trilice. 
Cecconello, A. M., Antoni, C. D. \& Koller, S. H. (2003). Práticas educativas, estilos parentais e abuso físico no contexto familiar. Psicologia em Estudo, 8 ( $\mathrm{n}^{\mathrm{o}}$ spe), 45-54.

Laranjeira, R., Pinsky, I., Zaleski, M. \& Caetano, R. (2007). I Levantamento Nacional Sobre os Padrões de Consumo de Álcool na População Brasileira. Brasília: SENAD (Secretaria Nacional Anti-Drogas).

Carlini, E. A., Galduróz, J. C. F., Noto, A. R. \& Nappo, A. S. (2005). II Levantamento domiciliar sobre o uso de drogas psicotrópicas no Brasil. São Paulo: CEBRID (Centro Brasileiro de Informações sobre Drogas Psicotrópicas)

Organização Mundial da Saúde [OMS] (2004). Neurociências: Consumo e dependência de substâncias psicoativas (resumo). Genebra : Autor.

Schenker, M., \& Minayo, M. C. S. (2003). A implicação da família no uso abusivo de drogas: uma revisão crítica. Ciência \& Saúde Coletiva, 8(1), 299-306.

Schenker, M. \& Minayo, M. C. S. (2005). Fatores de risco e de proteção para o uso de drogas na adolescência. Ciência \& Saúde Coletiva, 10(3), 707-717.

Duncan, S. C., Duncan, T. E., Biglan, A., \& Ary, D. (1998). Contributions of the social context to the development of adolescent substance use: a multivariate latent growth modeling approach. Drug and alcohol dependence, 50(1), 57-71.

Shucksmith, J., Glendinning, A., \& Hendry, L. (1997). Adolescent drinking behaviour and the role of family life: a Scottish perspective. Journal of Adolescence, 20(1), 85-101.

Jackson, C., Henriksen, L., Dickinson, D., Messer, L., \& Robertson, S. B. (1998). A longitudinal study predicting patterns of cigarette smoking in late childhoold. Health Education and Behavior, 25(4), 436-447.

Reifman, A., Barnes, G. M., Dintcheff, B. A., Farrel, M. P., \& Uhteg, L. (1998). Parental and peer influences on the onset of heavier drinking among adolescents. Journal of Studies on Alcohol, 59(3), 311-317.

Jackson, C., Henriksen, L., \& Dickinson, D. (1999). Alcohol-specific socialization, parenting behaviors and alcohol use by children. Journal of Studies on Alcohol, 60(3), 362-367.

Mott, J. Á, Crowe, P. A., Richardson, J., \& Flay, B. (1999). Afterschool supervision and adolescent cigarette smoking: contributions of seting and intensity of after-school self-care. Journal of Behavioral Medicine, 22(1), 35-58.

Baker, J. G., Rosenthal, S. L., Leonhardt, D., Kollar, L. M., Succop, P. A., Burklow, K. A., \& Biro, F. M. (1999). Relationship between perceived parental monitoring and young adolescent girls sexual and substance behaviors. Journal of Pediatric and Adolescent Gynecologic, 12(1), 17-22.

Li, X., Feigelman, S., \& Stanton, B. (2000). Perceived parental monitoring and health risk behaviors among urban low-income African-American children and adolescents. Jounal of Adolescence Health, 27(1),43-48.

Guo, J., Hawkins, J. D., Hill, K. G., \& Abbott, R. D. (2001). Childhood and adolescent predictors of alcohol abuse and dependence in young adulthood. Journal of Studies on Alcohol, 62(6), 754-762.

Raboteg-Saric, Z., Rijavec, M., \& Brajsa-Zganec, A. (2001). The relation of parental practices and self-conceptions to young adolescent problem behaviors and substance use. Nordic Journal of Psychiatry, 55(3), 203-209.

DiClemente, R. J., Wingood, G. M., Crosby, R., Sionean, C., Cobb, B. K., Harrington, K., Davies, S., Hook, E. W., \& Kim, M. (2001). Parental monitoring: association with adolescents risk behavior. Pediatrics, 107(6), 1363-1368.

Shope, J. T., Waller, P. F., Trivellore, E., Raghunathan, C. D., \& Patil, S. M. (2001). Adolescent antecedents of high-risk driving behavior into young adulthood: substance use and parental influences. Accident Analysis and Prevention, 33(1), 649-658.

Stanton, B., Li, X., Pack, R., Cottrel, L., Harris, C., \& Burns, J. M. (2002). Longitudinal influence of perceptions of peer and parental factors on African American adolescent risk involvement. Journal of Urban Health, 79(4), 536-548.

Simons-Mortons, B. G. (2002). Prospective analysis of peer and parent influences on smoking initiation among early adolescents. Prevention Science, 15(8), 111-115.

Simons-Morton, B. G., \& Haynie, D. L. (2003). Psychosocial predictors of increases smoking stage among sixth graders. American Journal of Health Promotion, 22(5), 125-137.

Rai, A. A., Stanton, B., Wu, Y., Li, X., Galbraith, J., Cottrell, L., Pack, R., Harris, C., Dálessadri, D., \& Burns, J. (2003). Relative influences of perceived parental monitoring and perceived peer involvement on adolescent risk behaviors: an analysis of six cross-sectional data sets. Jounal of Adolescent Health, 33(2), 108-118.

Borawski, E. A., Ievers-Landis, C. E., Lovegreen, L. D., \& Trapl, E. S. (2003). Parental monitoring, negotiated unsupervised time, and parental trust; the role of perceived parenting practices in adolescent health risk behaviors. Jounal of Adolescent Health, 33(2), 60-70.

Simons-Morton, B. G., Chen, R., Abroms, L., \& Haynie, D. L. (2004). Latent growth curve analyses of peer and parent influences on smoking progression among early adolescents. Health Psychology, 23(6), 612-621.

Simons-Morton, B. G. (2004). The protective effect of parental expectations against early adolescent smoking initiation. Health Education Research, 19(5) 561-569.

Wood, M. D., Read, J. P., Mitchell, R. E., \& Brand, N. H. (2004). Do parents still matter? Parent and peer influences on alcohol involvement among recent high school graduates. Psychology of Addictive Behaviors, 18(1), 19-30.

Ramirez, J. R., Crano, W. D., Quist, R., Burgoon, M., Alvaro, E. M., \& Grandpre, J. (2004). Acculturation, familism, parental monitoring, and knowledge as predictors of marijuana and inhlant use in adolescents. Psychology of Addictive Behaviors, 18(1), 311.

Wright, D. R., \& Fitzpatrick, K. M. (2004). Psychosocial correlates of substance use behaviors among African American youth. Adolescence, 39(156), 653-667.

Wills, T. A., Resko, J. A., Ainette, M. G., \& Mendoza, D. (2004). Role of Parent Support and Peer Support in Adolescent Substance Use: A Test of Mediated Effects. Psychology of Addictive Behaviors, 18(2), 122-134.

Bahr, S. J., Hoffmann, J. P., \& Yang, X. (2005). Parental and peer influences on the risk of adolescent drug use. The Journal of Primary Prevention, 26(6), 529-551. 
Hill, K. G., Hawkins, J. D., Catalano, R. F., Abbott, R. D., \& Guo, J. (2005). Family influences on the risk of daily smoking initiation. Jounal of Adolescent Health, 37(3),202-210.

Chuang, Y. C., Ennet, S. T., Bauman, K. E., \& Foshee, V. A. (2005). Neighborhood influences on adolescent cigarette and alcohol use: mediating effects throug parent and peer behaviors. Journal of Health and Social Behavior, 21(2), 29-41.

Clevend, M. G., Gibbons F. X., Gerrard, M., Pomery, E. A., \& Brody, G. H. (2005). The impact of parenting on risk cognitions and risk behavior: a study of mediation and moderation in a panel of African American Adolescents. Child Development,76(4), 900916.

Nash, S. G., McQueen, A., \& Bray, J. H. (2005). Pathways to adolescent alcohol use: family environment, peer influence, and parental expectations. Jounal of Adolescent Health, 37(1), 19-28.
Simons-Morton, B. G., \& Chen, R. (2005). Latent growth curve analyses of parent influences on drinking progression among early adolescents. Journal of Studies on Alcohol, 66(1), 5-13.

Springer, A. E., Sharma, S., Guardado, A. M., Nava, F. V., \& Kelder, S. H. (2006). Perceived parental monitoring and health risk behavior among public secondary school students in El Salvador. The Scientific World Journal, 6(3), 1810-1814.

Siebenbruner, J., Englund, M. M., Egeland, B., \& Hudsons, K. (2006). Developmental antecedents of late adolescence substance use patterns. Development and Psychopathology, 26(2), 95-101.

Stattin, H., \& Kerr, M. (2000). Parental monitoring: A reinterpretation. Child Development, 71(4), 1072-1085.

Recebido em 18/03/2008 Aceito em 23/07/2008

Endereço para correspondência : Telmo Mota RonzaniRua Ministro Amarílio Lopes Salgado, 36/101, Cascatinha, CEP: 36033290, Juiz de Fora-MG, Brasil. E-mail: telmo.ronzani@ufjf.edu.br 